\title{
Existence and nonlinear stability of convective solutions for almost compressible fluids in Bénard problem
}

\author{
Antonino De Martino * Arianna Passerini ${ }^{\dagger}$
}

October 29, 2019

\begin{abstract}
We study the nonlinear almost compressible 2D Oberbeck-Boussinesq system, characterized by an extra buoyancy term where the density depends on the pressure, and a corresponding dimensionless parameter $\beta$, proportional to the (positive) compressibility factor $\beta_{0}$. The local in time existence of the perturbation to the conductive solution is proved for any "size" of the initial data. However, unlike the classical problem where $\beta_{0}=0$, a smallness condition on the initial data is needed for global in time existence, along with smallness of the Rayleigh number. Removing this condition appears quite challenging and we leave it as an open question.
\end{abstract}

\section{Introduction}

In modeling convective phenomena, almost all available results of existence, stability etc., are achieved in the Oberbeck-Boussinesq (O-B) approximation $[1,8,11,18,15,17,7]$. As is well known, in spite of the need of compressibility to produce convection, the peculiarity of this approximation consists in keeping the incompressibility hypothesis $\nabla \cdot \vec{v}=0$ (with $\vec{v}$ velocity field of the fluid), while allowing for (linear) variation of density with temperature only in the term involving the external force (gravity). It must be noted that the O-B model is pretty accurate even for gas flow, though the corresponding instability prediction occurs at a threshold lower than that suggested by experiments.

However, in the recent paper [9] it was shown that the O-B model presents some basic drawbacks. In the first place, it was noticed that any change in the density would be at odds with the Gibbs law, if dependence on $p$ was not taken into account; in addition, such a dependence was also necessary in order to avoid instability in wave propagation.

Thus, in order to make the O-B model more "natural" and accurate, in [9], among other things, it was proposed a rather general approach, consistent with thermodynamical principles, that includes an extra buoyancy term depending on the pressure field. In other words, the variation of the density, $\rho$ in the gravity force has the following expression

$$
\rho=\rho_{0}\left(1-\alpha_{0}\left(T-T_{0}\right)+\beta_{0}\left(p-p_{0}\right)\right)
$$

\footnotetext{
* Dipartimento di Matematica, Politecnico di Milano, Via Bonardi 9,20133 Milano, Italy, e-mail: antonino.demartino@polimi.it

${ }^{\dagger}$ Department of Mathematics and Computer Science, University of Ferrara, Via Machiavelli 30, 40121 Ferrara, Italy, e-mail: ari@unife.it
} 
where $T, p$, are temperature and pressure fields, Moreover, $\rho_{0}, T_{0}$ and $p_{0}$ are (constant) gauge density, temperature and pressure while $\alpha_{0}$ and $\beta_{0}$ are respectively the thermal expansion coefficient and the compressibility. We assume they are positive constants depending on the material. The classical O-B approximation is then re-obtained by setting $\beta_{0}=0$.

One important consequence of this more comprehensive scheme is that the well-posedness of the associated mathematical problem requires now the pressure field to be treated as an independent unknown, satisfying a suitable elliptic problem and subject to Robin boundary conditions, see [3]

$$
\nabla p \cdot \vec{n}+\beta_{0} p=0, \quad \text { on } \quad \partial \Omega,
$$

where $\vec{n}$ is the outer normal.

It is just this feature that makes the problem particularly interesting and more difficult than the classical one.

In order to test some significant aspects of the generalized model, in the joint paper [16], the second author has investigated its stability predictions in the simple physical setting of the classical Bénard problem of a horizontal layer of fluid heated from below. Here, the basic state $\mathrm{s}_{0}=(\vec{v}, T, p)_{0}$, consists of the fluid at rest, subject to a constant temperature gradient and a suitable corresponding pressure distribution. More precisely, denoting by $z$ the vertical coordinate, $h$ the thickness of the layer, $g$ the gravity, and $T_{0}$ and $T_{0}-\delta T<T_{0}$ the temperatures at the bottom and the top of the layer, we have that $\mathrm{s}_{0}$ is characterized by

$$
\begin{gathered}
\vec{v} \equiv 0, \quad T(z)=T_{0}-\frac{\delta T}{h} z \\
p(z)=p_{0}+p_{b} e^{-\rho_{0} g \beta_{0} z}+\frac{1}{\beta_{0}^{2}} \frac{\alpha_{0} \delta T}{\rho_{0} g h}\left(1-e^{-\rho_{0} g \beta_{0} z}\right)-\frac{1}{\beta_{0}}\left(\frac{\alpha_{0} \delta T}{h} z+1-e^{-\rho_{0} g \beta_{0} z}\right),
\end{gathered}
$$

where the constant $p_{0}$ is a gauge pressure, while $p_{b}$ is a prescribed value arising from the appropriate boundary condition on $p$. Notice that this basic solution is peculiar of the new model and reduces to that of the $\mathrm{O}-\mathrm{B}$ one formally taking $\beta_{0}$ : the first bracket tends asymptotically to the first term of the second bracket. The main result proved in [3] states that, in the linear stability framework, convective rolls set in at a Rayleygh number (basically, temperature gradient) less than the critical value predicted by the classical model. This result is shown under "stress-free" boundary conditions on $\vec{v}$, which we shall also adopt throughout this paper.

The main objective of this article is to perform a nonlinear analysis of the stability of the basic flow (1.1) and, as a necessary requirement, to investigate the well-posedness of the associated initial-boundary value problem for the perturbation fields. We shall be concerned with 2D perturbations. The reason of this choice is because, unlike the analogous problem for the classical O-B model, this case already presents an unusual feature. More precisely, the proof of global existence of strong solutions can be seemingly obtained only by restricting the size of the energy -namely, the $L^{2}$-norm- of the initial data (and, of course, of the relevant dimensionless parameters). As a matter of fact, even the existence of weak solutions appears difficult to establish, because of lack of a uniform bound of the energy on a time interval of arbitrary length. The reason for this unexpected, somehow, situation is due to the fact, mentioned earlier on, that the pressure is now an independent unknown satisfying a suitable elliptic problem under Robin boundary conditions. More importantly, it enters the linear momentum equation no longer just in a gradient form (see $\left.(2.1)_{2}\right)$. We leave it as an interesting open question whether global weak and/or strong solutions can be obtained without restricting the size of the initial data. In fact, it would be of some interest to find out whether smallness of the initial energy is just a mathematical requirement, or else a necessary physical property. 
It is worth remarking that the extension of our result to the 3D case, under analogous restrictions on the initial data, does not present conceptual difficulties and will be treated elsewhere $[14]$.

The plan of the paper is as follows: in Section 2 we formulate the nonlinear stability problem, moreover, we recall some previous findings and prove a number of basic properties for the pressure field. In particular, we show unique solvability for the pressure equation, on condition that the material constant $\beta$ is less than $2 \pi$. Notice that such a restriction was also needed in the linear analysis [3]. In the subsequent Section 3 we devote our effort to the proof of existence for the nonlinear perturbation problem. We exploit the usual techniques of functional analysis applied to the study of this type of questions $[2,7,12,13]$. In particular, we derive several a priori "energy" estimates and couple them with the classical Gälerkin method with a special basis, to prove existence of solutions. For all Rayleigh numbers and initial data of arbitrary "size", it is shown that a strong solution (in the sense of Ladyzhenskaya; see e.g. [12]) does exist for some time interval $[0, T)$ where $T$ can be estimated in terms of the initial data. However, if only the $L^{2}$-norm of these data is sufficiently small and the Rayleigh number is below a certain constant, then we can take $T=\infty$. Furthermore, we show that, in such a case, all solutions must decay to zero, as time increases, exponentially fast, thus also proving the nonlinear stability of the state (1.1).

\section{Preliminary results}

We begin to introduce some notation. We recall that the layer is bounded in the $z$ (vertical) direction and unbounded and invariant in the $x$-direction. The observed convective rolls are also invariant in the $y$-direction and can be described by $x$-periodic functions. Then, the relevant region of flow can be written in non-dimensional variables as

$$
\Omega:=\left\{(x, y, z) \in \mathbb{R}^{3}: z \in(0,1)\right\} .
$$

Since we are interested in $2 \mathrm{D}$ flow, we restrict the spatial domain to the periodicity cell $\Omega_{0}=$ $\{(x, z) \in(0,1) \times(0,1)\}$.

Definition 2.1. (Mean value of $f$ )

$$
\langle f\rangle=\frac{1}{\left|\Omega_{0}\right|} \int_{\Omega_{0}} f(x, z, t) d x d z
$$

For the derivates we write $\partial_{\xi} \phi:=\frac{\partial \phi}{\partial \xi}\left(:=\phi_{\xi}\right)$ for any variable $\xi \in\{x, z, t\}$ and analogously for higher order derivates. We denote the material derivate by $\frac{d}{d t}$ or by a superposed dot. The starting point of our analysis is the (nondimensional) system governing the evolution of the perturbation $(\vec{v}, \tau, P)$ to the basic state $(1.1)$, see [3, pag. 5]:

$$
\left\{\begin{array}{l}
\nabla \cdot \vec{v}=0 \\
\frac{1}{\operatorname{Pr}}\left(\frac{\partial \vec{v}}{\partial t}+\vec{v} \cdot \nabla \vec{v}\right)=-\nabla P-\beta P \vec{k}+\Delta \vec{v}+\operatorname{Ra} \tau \vec{k}, \\
\frac{\partial \tau}{\partial t}+\vec{v} \cdot \nabla \tau-\Delta \tau=\vec{v} \cdot \vec{k}
\end{array}\right.
$$

where $\vec{k}$ is the upward unit vector and $P=p-p(z)$, with $p(z)$ given in (1.1). Moreover, $\operatorname{Pr}:=\frac{\nu}{D}$ and Ra $:=\frac{\alpha_{0} g \delta T h^{3}}{\nu D}$ are Prandtl and Rayleigh numbers, $D$ is the diffusivity, while $\beta:=\rho_{0} g \beta_{0} h$. We endow the above system with stress-free boundary conditions for the velocity field $\vec{v}$ and 
(homogeneous) Dirichlet boundary condition for $\tau$. Thus, we obtain the following set of side requirements

$$
\begin{gathered}
v^{z}(x, 0, t)=v^{z}(x, 1, t)=0, \\
v_{z}^{x}(x, 0, t)=v_{z}^{x}(x, 1, t)=0, \\
\tau(x, 0, t)=\tau(x, 1, t)=0 .
\end{gathered}
$$

By taking the divergence of $(2.1)_{2}$ one necessarily get an equation for $P$ as unknown

$$
\Delta P+\beta P_{z}=-\frac{1}{\operatorname{Pr}} \nabla \cdot(\vec{v} \cdot \nabla \vec{v})+\operatorname{Ra} \tau_{z}
$$

with Robin's boundary conditions

$$
P_{z}(x, 0, t)+\beta P(x, 0, t)=P_{z}(x, 1, t)+\beta P(x, 1, t)=0 .
$$

Herein, we are going to show that the existence of a solution $P$ for this problem is also sufficient to solve (2.1). To this end, as done in [3], we set $\Pi=P e^{\beta z}$ and face the system

$$
\left\{\begin{array}{l}
\Delta \Pi-\beta \Pi_{z}=-\frac{1}{\operatorname{Pr}} e^{\beta z} \nabla \cdot(\vec{v} \cdot \nabla \vec{v})+\operatorname{Ra} e^{\beta z} \tau_{z}, \\
\frac{1}{\operatorname{Pr}}\left(\frac{\partial \vec{v}}{\partial t}+\vec{v} \cdot \nabla \vec{v}\right)-\Delta \vec{v}=-\nabla\left(e^{-\beta z} \Pi\right)-\beta e^{-\beta z} \Pi \vec{k}+\operatorname{Ra} \tau \vec{k}, \\
\frac{\partial \tau}{\partial t}+\vec{v} \cdot \nabla \tau-\Delta \tau=\vec{v} \cdot \vec{k}
\end{array}\right.
$$

still with conditions (2.9), (2.2), (2.4) and with Neumann conditions for $\Pi$ on $\partial \Omega$ :

$$
\Pi_{z}(x, 0, t)=\Pi_{z}(x, 1, t)=0 .
$$

We underline that one can not get rid of (2.5) in the present context, which is a full coupling context, so that (2.5) has to be inserted in system (2.1). Notice that $t$ is just a parameter for the elliptic problem given by $(2.7)_{1}$ and $(2.8)$ for data $(\vec{v}, \tau)$.

In addition, we shall assume periodicity in the $x$-direction. Finally, we append the initial conditions:

$$
(\vec{v}(x, z, 0), \tau(x, z, 0))=\left(\vec{v}_{0}(x, z), \tau_{0}(x, z)\right) .
$$

We are going to prove the existence for $\Pi$ in

$$
\mathbb{F}:=\left\{p \in L^{2}\left(\Omega_{0}\right):\langle p\rangle=0, \quad \text { periodic in } \quad x, \quad \partial_{z} \Pi=0 \quad \text { in } \quad z=0,1\right\} .
$$

In $\mathbb{F}$ for $(m, n) \in \mathbb{N}_{0} \times \mathbb{N}_{0}$ we have the basis

$$
\phi_{m n}^{i}(x, z)= \begin{cases}\cos (2 \pi m x) \cos (\pi n z) & \text { if } \quad i=1, \\ \sin (2 \pi m x) \cos (\pi n z) & \text { if } \quad i=-1 .\end{cases}
$$

Remark 2.1. All the functions in this basis have mean value zero.

For the "temperature" field $\tau$ and the stream function $\varphi$, associated to $\vec{v}$ by

$$
v^{x}=-\varphi_{z} \quad v^{z}=\varphi_{x}
$$

we can use

$$
\xi_{m n}^{i}(x, z)= \begin{cases}\cos (2 \pi m x) \sin (\pi n z) & \text { if } \quad i=1 \\ \sin (2 \pi m x) \sin (\pi n z) & \text { if } \quad i=-1\end{cases}
$$


In the periodicity cell, which is bounded, we use the Lebesgue and Sobolev spaces which are denoted by $L^{q}$ (with the norm $\|\cdot\|_{q}$ ) and $W^{l, q}$ (with the norm $\|\cdot\|_{l, q}$ ).

We construct Sobolev spaces starting from bases (2.10) and (2.11). We define $W_{N}^{k, 2}\left(\Omega_{0}\right)$, $k=0,1,2$ as the closure with respect to the $W^{k, 2}\left(\Omega_{0}\right)$-norm of finite combinations of elements of the basis (2.10). We denote by $W_{D}^{k, 2}$ the subspace generated by the closure of the linear hull of (2.11) in the $W^{k, 2}\left(\Omega_{0}\right)$-norm, for $k=0,1,2$. Analogously, we denote by $\mathcal{W}_{D}^{k, 2}\left(\Omega_{0}\right)$ with $k=0,1,2$ the linear hull of of the vectorial divergence free functions obtained from (2.11) taken as stream functions, and afterwards closed by the $W^{k, 2}\left(\Omega_{0}\right)$-norm for $k=0,1,2$. If $k=0$ we denote these spaces as $H_{D}\left(\Omega_{0}\right)$ and $\mathcal{H}_{D}\left(\Omega_{0}\right)$, respectively.

As customary, in $L^{2}\left(\Omega_{0}\right)$ we use the scalar product

$$
(u, v):=\int_{\Omega_{0}} u v d x d z .
$$

We recall the Bochner spaces $L^{q}\left((0, T) ; W^{m, p}\left(\Omega_{0}\right)\right)$, i.e. $L^{q}$ functions on the interval $(0, T)$ defined in the Sobolev space $W^{m, p}\left(\Omega_{0}\right)$ with norm

$$
\begin{aligned}
|u|_{q, m, p} & :=\left(\int_{0}^{T}\|u(t)\|_{m, p}^{q} d x\right)^{\frac{1}{q}}, \\
|u|_{\infty, m, p} & :=e s s \sup _{t \in[0, T]}\|u(t)\|_{m, p} .
\end{aligned}
$$

We also recall some inequalities, holding true in $\Omega_{0}$ : the Poincaré inequality ([3, pag. 12]) for $\Pi$ :

$$
\|\Pi\|_{2} \leq \frac{1}{2 \pi}\|\nabla \Pi\|_{2}
$$

the Poincaré inequality for the vectorial field $\vec{v}$, which can be found in [5],

$$
\|\vec{v}\|_{2} \leq\|\nabla \vec{v}\|_{2}
$$

and for $\tau$ (see [3]):

$$
\|\tau\|_{2} \leq \frac{1}{\sqrt{5} \pi}\|\nabla \tau\|_{2}
$$

Moreover, in order to deal with the nonlinearities in two dimensions, use will be made of Ladyzhenskaya's inequality (see [5]):

$$
\|\vec{v}\|_{4} \leq\left(\frac{1}{\sqrt{2}}\right)^{\frac{1}{2}}\|\vec{v}\|_{2}^{\frac{1}{2}}\|\nabla \vec{v}\|_{2}^{\frac{1}{2}}
$$

Moreover, we know that the norms of $\partial_{x x}, \partial_{y y}$ are equivalent to the full set of the second derivatives. In particular

$$
\frac{1}{16}\|\Delta \vec{v}\|_{2} \leq\left\|D^{2} \vec{v}\right\|_{2} \leq \frac{1}{4}\|\Delta \vec{v}\|_{2}
$$

and the same for $\tau$. The proof can be found in [3].

Still in [3], the reduced system

$$
\left\{\begin{array}{l}
\Delta \Pi-\beta \Pi_{z}=e^{\beta z} f \quad \text { in } \quad \Omega_{0} \\
\Pi_{z}(x, 0)=\Pi_{z}(x, 1)=0 \quad \text { for } \quad x \in \mathbb{R}
\end{array}\right.
$$

allows for zero mean value periodic solutions in $W_{N}^{2,2}\left(\Omega_{0}\right)$ if $\langle f\rangle=0$, in this way the linear differential operator is invertible. We give here a shorter proof under more general conditions than in [3]. 
Theorem 2.1. Let $f \in L^{2}\left(\Omega_{0}\right)$, and assume $0 \leq \beta<2 \pi$. Then problem (2.13) with homogeneous Neumann conditions has a unique $x$ - periodic solution $\Pi \in W^{2,2}\left(\Omega_{0}\right)$ such that $\langle\Pi\rangle=0$ and the following estimates hold true

$$
\|\nabla \Pi\|_{2} \leq \frac{1}{2 \pi-\beta}\left\|e^{\beta z} f\right\|_{2}, \quad \quad\|\Delta \Pi\|_{2} \leq \frac{2 \pi}{2 \pi-\beta}\left\|e^{\beta z} f\right\|_{2} .
$$

Proof. We introduce the test function space

$$
H:=\left\{\varphi \in W^{1,2}\left(\Omega_{0}\right): \int_{\Omega_{0}} \varphi=0\right\} .
$$

By multiplying both sides $(2.13)_{1}$ by $\varphi \in H$, integrating by parts over $\Omega$ (x-periodicity) and taking into accounts the boundary conditions, we formally obtain

$$
a(\Pi, \varphi):=(\nabla \Pi, \nabla \varphi)+\beta\left(\Pi_{z}, \varphi\right)=-\left(e^{\hat{\beta} z} f, \varphi\right) \text { for all } \varphi \in H .
$$

This is a weak form of $(2.13)$ and $a(\Pi, \varphi)$ is a bounded bilinear form because

$$
(\nabla \Pi, \nabla \varphi)+\beta\left(\Pi_{z}, \varphi\right) \leq\|\nabla \Pi\|_{2}\|\nabla \varphi\|_{2}+\beta\|\nabla \Pi\|_{2}\|\varphi\| \leq C\|\Pi\|_{1,2}\|\varphi\|_{1,2} .
$$

Furthermore by Cauchy-Schwarz and Poincaré inequalities

$$
\begin{aligned}
a(\Pi, \Pi) & =(\nabla \Pi, \nabla \Pi)+\beta\left(\Pi_{z}, \Pi\right) \geq(\nabla \Pi, \nabla \Pi)-\beta\left(\Pi_{z}, \Pi\right) \\
& \geq\|\nabla \Pi\|_{2}^{2}-\frac{\beta}{2 \pi}\|\nabla \Pi\|_{2}^{2}=\left(1-\frac{\beta}{2 \pi}\right)\|\nabla \Pi\|_{2}^{2} .
\end{aligned}
$$

Existence and uniqueness then come from (2.16) by the Lax-Milgram theorem if $\beta<2 \pi$.

In Section 3, to construct the solution of the full system, we need an explicit expression of the solution $\Pi$ of problem (2.13) and we can use the corresponding coefficients of the basis functions (2.10) as given in [3], since the data verify the condition $\langle f\rangle=0$. Actually, the property holds true because if the data of (2.13) are taken from the full system, then from the boundary conditions one sees

$$
\langle f\rangle=-\frac{1}{\operatorname{Pr}}\langle\nabla \cdot(\vec{v} \cdot \nabla \vec{v})\rangle+\operatorname{Ra}\left\langle\tau_{z}\right\rangle=0 .
$$

Now, we prove a further estimate:

Lemma 2.1. Assume $\beta<2 \pi$ and let $\Pi \in W^{2,2}\left(\Omega_{0}\right)$ be the unique zero mean value solution of

$$
\Delta \Pi-\beta \Pi_{z}=e^{\beta z} \nabla \cdot \vec{w},
$$

with Neumann conditions at $z=0,1$ and $x$-periodic conditions: if $\vec{w} \cdot \vec{n}=0$ and $\vec{w} \in W^{1,2}\left(\Omega_{0}\right)$, then

$$
\|\Pi\|_{2} \leq c(\beta)\|\vec{w}\|_{2} .
$$

where $c(\beta)$ is a constant increasing with $\beta$ and bounded from below.

Proof. We choose test functions such that

$$
\left(\Delta \Pi-\beta \Pi_{z}, \psi\right)=\left(\Pi, \Delta \psi+\beta \psi_{z}\right) .
$$


Actually,

$$
\begin{aligned}
& (\Delta \Pi, \psi)-\beta\left(\Pi_{z}, \psi\right)= \\
& \int_{\Omega_{0}}\left[\nabla \cdot(\psi \nabla \Pi-\Pi \nabla \psi)-\beta \frac{\partial(\Pi \psi)}{\partial z}\right]+(\Pi, \Delta \psi)+\beta\left(\Pi, \psi_{z}\right) .
\end{aligned}
$$

Once the Neumann conditions on $\Pi$ are stated, then the boundary term vanishes in two cases: if $\psi$ verify Robin's conditions

$$
\nabla \psi \cdot \vec{n}+\beta \psi=0, \quad z=0,1
$$

on both boundaries, and if $\psi$ is constant, this last possibility is irrelevant: actually if we want to estimate the $L^{2}$-norm of a zero mean value function by the Riesz theorem, then constant test functions give no contribution, since they lie in the kernel of the functional.

Therefore, we just need to show that $R(\beta)>0$ exists such that

$$
\|\Pi\|_{2}=\sup _{\|\varphi\|_{2} \leq 1}|(\Pi, \varphi)|=\sup _{\|\varphi\|_{2} \leq 1}|(\Pi, \varphi-\langle\varphi\rangle)|=\sup _{\|\psi\|_{2,2} \leq R}\left|\left(\Pi, \Delta \psi+\beta \psi_{z}\right)\right| .
$$

because $(\Pi,\langle\varphi\rangle)=\langle\varphi\rangle\langle\Pi\rangle=0$. So, let us look for $\psi$ verifying $\Delta \psi+\beta \psi_{z}:=\varphi-\langle\varphi\rangle$ with Robin's boundary condition. If we make the substitution $\bar{\psi}=e^{-\beta z} \psi$, by [3] the equation above with Robin's condition is equivalent to (2.13) with Neumann conditions. So, from Theorem $2.1 \mathrm{a}$ mean value zero solution $\bar{\psi}$ exists and is unique. In this way we proved (2.23): for all $\varphi$ we can write $\psi=e^{\beta z} \bar{\psi}$.

Now, we want to estimate the left hand side of (2.20) by the right-hand side of equation (2.18) tested by $\psi \in W^{2,2}\left(\Omega_{0}\right)$

$$
\begin{aligned}
\left(\Delta \Pi-\beta \Pi_{z}, \psi\right) & =\left(\nabla \cdot\left(e^{\beta z} \vec{w}\right)-\beta e^{\beta z} w^{z}, \psi\right) \\
& =\int_{\Omega_{0}} \nabla \cdot\left(\psi e^{\beta z} \vec{w}\right) d \Omega_{0}-\left(e^{\beta z} \vec{w}, \nabla \psi\right)-\left(\beta e^{\beta z} w^{z}, \psi\right) . \\
& \leq e^{\beta}(1+\beta)\|\vec{w}\|_{2}\|\psi\|_{1,2} .
\end{aligned}
$$

Here, the boundary term vanishes by the hypothesis on $\vec{w}$.

Finally, we can write

$$
\|\Pi\|_{2}=\sup _{\|\psi\|_{2,2} \leq R}\left|\left(\Pi, \Delta \psi+\beta \psi_{z}\right)\right| \leq \sup _{\|\psi\|_{2,2} \leq R} e^{\beta}(1+\beta)\|\vec{w}\|_{2}\|\psi\|_{1,2} \leq e^{\beta}(1+\beta) R\|\vec{w}\|_{2}
$$

Remark 2.2. If the right hand side of (2.18) contains terms of the kind $\vec{w}=\vec{v} \cdot \nabla \vec{v}$ with stressfree and impermeability conditions on $\vec{v}$ (besides of the periodicity in $x$ ), their inner product with $\psi \in W^{2,2}\left(\Omega_{0}\right)$ allows several applications of the Gauss theorem with boundary integrals each time vanishing. One easily sees

$$
\begin{gathered}
\left.\int_{\Omega_{0}} e^{\beta z} \nabla \cdot(\vec{v} \cdot \nabla \vec{v}) \psi=-\int_{\Omega_{0}} e^{\beta z} \beta \nabla \cdot\left(v^{z} \vec{v}\right) \psi+\nabla \cdot(\vec{v} \otimes \vec{v}) \cdot \nabla \psi\right]= \\
=\int_{\Omega_{0}} e^{\beta z}\left[\beta\left(v^{z}\right)^{2} \psi+2 \beta v^{z} \vec{v} \cdot \nabla \psi+\vec{v} \cdot\left(D^{2} \psi\right) \cdot \vec{v}\right] \\
\leq e^{\beta}(\beta+1)^{2}\|\vec{v}\|_{4}^{2}\|\psi\|_{2,2} .
\end{gathered}
$$


The estimate so obtained is the generalization of the classical one

$$
\|p\|_{2} \leq c\|\vec{v}\|_{4}^{2}
$$

holding at $\beta=0$ for the classical $O-B$ approximation. This is due to the particular boundary conditions and the particular domain (bounded in one direction and with flat boundaries).

Finally, estimate (2.14) too can slightly be changed by considering $f=\nabla \cdot \vec{w}$ with $\vec{w} \cdot \vec{n}=0$ at the boundary:

$$
\|\nabla \Pi\|_{2} \leq \frac{c(\beta)}{2 \pi-\beta}\|\vec{w}\|_{2}
$$

Remark 2.3. In [3] it was observed that system (2.1) with the boundary conditions (2.2) - (2.3) is satisfied if $\vec{v}=c \vec{i}, \tau=0$ and $\Pi=0$, so that for all $c>0$ it is the basic solution. The constant $c$ is an arbitrary real number, so that this class of solutions corresponds to the Galileian invariance of the equation, and we can call it null solution (though it is not exactly the rest state). In fact, if we define a subspace by the condition $\Pi_{x}=0, \vec{v}_{x}=0$ and $\tau_{x}=0$, the full-system projects in such subspace since it becomes linear. For any size of the dimensionless parameters and of the initial data, one can find particular solutions of $(2.1)$ in the form $(\Pi, \vec{v}, \tau)=$ $(\mathcal{G}(z, t), \mathcal{A}(z, t) \vec{i}, \mathcal{T}(z, t))$. Such solutions have to verify:

$$
\left\{\begin{array}{l}
\frac{\partial^{2} \mathcal{G}}{\partial z^{2}}-\beta \frac{\partial \mathcal{G}}{\partial z}=\operatorname{Ra} e^{\beta z} \frac{\partial \mathcal{I}}{\partial z} \\
\frac{1}{\operatorname{Pr}} \frac{\partial \mathcal{A}}{\partial t}-\frac{d^{2} \mathcal{A}}{\mathcal{J} z^{2}}=0 \\
\frac{\partial \mathcal{J}}{\partial t}-\frac{\partial^{2} \mathcal{J}}{\partial z^{2}}=0 .
\end{array}\right.
$$

Actually, for any $\mathrm{Ra}, \operatorname{Pr}$ and $\beta$ positive, problem (2.1) with arbitrary $f, g \in W^{m, 2}(0,1)$ and $m \in \mathbb{N}$

has a unique global solution

$$
\left\{\begin{array}{l}
v^{x}(x, z, 0)=f(z) \\
v^{z}(x, z, 0)=0 \\
\tau(x, z, 0)=g(z)
\end{array}\right.
$$

$$
\begin{aligned}
& \vec{v} \in L^{\infty}\left((0, \infty) ; W^{m, 2}(0,1)\right) \\
& \tau \in L^{2}\left((0, \infty) ; W^{m, 2}(0,1)\right) .
\end{aligned}
$$

Such solutions are clearly

$$
\mathcal{A}=2 \sum_{n=1}^{\infty}\left(\int_{0}^{1} f(s) \cos (n \pi s) d s\right) \cos (n \pi z) e^{-\operatorname{Pr} n^{2} \pi^{2} t}
$$

For $\mathcal{T}$ and $\mathcal{G}$ we can write as in [16]

$$
\begin{gathered}
\mathcal{T}=2 \sum_{n=1}^{\infty}\left(\int_{0}^{1} g(s) \sin (n \pi s) d s\right) \sin (n \pi z) e^{-n^{2} \pi^{2} t} . \\
\mathcal{G}=e^{-\beta z} \psi, \quad \text { where } \frac{\partial \psi}{\partial z}=\operatorname{Ra} e^{\beta z} \mathcal{T},
\end{gathered}
$$

so that

$$
\mathcal{G}=\mathcal{G}_{0}+2 \operatorname{Ra} \sum_{n=1}^{\infty} \frac{e^{-n^{2} \pi^{2} t}}{n^{2} \pi^{2}+\beta^{2}}\left(\int_{0}^{1} g(s) \sin (n \pi s) d s\right)\left(\beta \sin (n \pi z)+n \pi\left(e^{-\beta z}-\cos n \pi z\right)\right),
$$

where $\mathcal{G}_{0}$ is a constant. 


\section{Basic apriori estimates}

First a priori estimate

This estimate leads, as we prove in the next section, to a solution such that

$$
\begin{gathered}
\Pi \in L^{2}\left(0, T ; L^{2}(0, T)\right), \\
\vec{v} \in L^{\infty}\left(0, T ; \mathcal{H}\left(\Omega_{0}\right)\right) \cap L^{2}\left(0, T ; \mathcal{W}_{D}^{1,2}\left(\Omega_{0}\right)\right), \\
\tau \in L^{\infty}\left(0, T ; H\left(\Omega_{0}\right)\right) \cap L^{2}\left(0, T ; W_{D}^{1,2}\left(\Omega_{0}\right)\right),
\end{gathered}
$$

for bounded $\mathrm{T}$ which becomes unbounded as $\beta$ goes to 0 .

From the estimate (2.19) and by the Remark 2.2 we have

$$
\|\Pi\|_{2} \leq C(\beta)\left[\frac{1}{\operatorname{Pr}}\|\vec{v}\|_{4}^{2}+\operatorname{Ra}\|\tau\|_{2}\right] .
$$

where we denote by $C(\beta)$ any, possibly different, function of $\beta$ such that $\lim _{\beta \rightarrow 0} C(\beta)=c \in \mathbb{R}_{+}$.

As customary, we introduce the energy:

$$
E(t):=\frac{1}{2}\left(\frac{\|\vec{v}\|_{2}^{2}}{\operatorname{Pr}}+\operatorname{Ra}\|\tau\|_{2}^{2}\right) .
$$

Now, we formally test $(2.1)$ with $(\vec{v}, \tau)$, then we multiply the third equation by Ra, we use Ladyzenskaya and Poincaré inequalities and sum

$$
\begin{aligned}
\frac{d}{d t} E(t)+\|\nabla \vec{v}\|_{2}^{2}+\operatorname{Ra}\|\nabla \tau\|_{2}^{2} & =-\beta\left(e^{-\beta z} \Pi, v^{z}\right)+2 \operatorname{Ra}\left(\tau, v^{z}\right) \\
& \leq \beta\|\Pi\|_{2}\|\vec{v}\|_{2}+2 \operatorname{Ra}\|\tau\|_{2}\|\vec{v}\|_{2} \\
& \leq \frac{\beta C(\beta)}{\operatorname{Pr}}\|\vec{v}\|_{4}^{2}\|\vec{v}\|_{2}+\operatorname{Ra}(\beta C(\beta)+2)\|\tau\|_{2}\|\vec{v}\|_{2} \\
& \leq \frac{\beta^{2} C^{2}(\beta)}{2 \operatorname{Pr}^{2}}\|\vec{v}\|_{2}^{4}+\frac{\|\nabla \vec{v}\|_{2}^{2}}{2}+(\beta C(\beta)+2) \operatorname{Ra}\left(\frac{\|\vec{v}\|_{2}^{2}}{2}+\frac{\|\tau\|_{2}^{2}}{2}\right)
\end{aligned}
$$

So that finally

$$
\frac{d E(t)}{d t}+\frac{\|\nabla \vec{v}\|_{2}^{2}}{2}+\operatorname{Ra}\|\nabla \tau\|_{2}^{2} \leq \mathbf{C}\left(E^{2}(t)+E(t)\right)
$$

where

$$
\mathbf{C}=\max \left\{\beta^{2} C^{2}(\beta),(\beta C(\beta)+2) \max \{\operatorname{Pr} \operatorname{Ra}, 1\}\right\} .
$$

Now, choosing a positive $\epsilon<1$ and disregarding the positive definite functions on the left hand side, we can integrate the inequality and the outcome is

$$
\frac{E(t)(E(0)+1)}{E(0)(E(t)+1)} \leq e^{\mathrm{C} t}
$$

It follows

$$
\begin{gathered}
1-e^{\mathbf{C} t} \frac{E(0)}{E(0)+1} \leq \frac{1}{E(t)+1} . \\
E(t) \leq \frac{e^{\mathbf{C} t} E(0)}{1+E(0)\left(1-e^{\mathbf{C} t}\right)} \quad \forall t<2 T:=\log \left[\left(\frac{E(0)+1}{E(0)}\right)\right]^{1 / \mathbf{C}} .
\end{gathered}
$$


Now, $E(t)$ is bounded as follows

$$
E(t) \leq \frac{\sqrt{E(0)(E(0)+1)}}{1+E(0)-\sqrt{E(0)(E(0)+1)}}:=\mathcal{M}(E(0)) \quad \forall t \in[0, T] .
$$

Then, by inserting (3.5) in (3.2) and integrating in $[0, T]$

$$
\int_{0}^{T}\left(\|\nabla \vec{v}\|_{2}^{2}+\|\nabla \tau\|_{2}^{2}\right) d t<\frac{2 \mathbf{C}}{\min \{\mathrm{Ra}, 1 / 2\}}\left(\mathcal{M}^{2}+\mathcal{M}\right) \log \left[\left(\frac{E(0)+1}{E(0)}\right)\right]^{\frac{1}{2 \mathrm{C}}}+E(0):=\mathcal{M}_{1},
$$

where we put $T$ as defined in (3.4), $\mathcal{M}$ is as in (3.5) and tends to 0 if $E(0)$ does.

\section{Second a priori estimate}

This estimate gives further regularity to any solution verifying the first one. In particular

$$
\begin{gathered}
\Pi \in L^{2}\left((0, T) ; L^{2}(0, T)\right), \\
\vec{v} \in L^{\infty}\left((0, T) ; \mathcal{W}_{D}^{1,2}\left(\Omega_{0}\right)\right) \cap L^{2}\left((0, T) ; \mathcal{W}_{D}^{2,2}\left(\Omega_{0}\right)\right), \\
\tau \in L^{\infty}\left((0, T) ; W_{D}^{1,2}\left(\Omega_{0}\right)\right) \cap L^{2}\left((0, T) ; W_{D}^{2,2}\left(\Omega_{0}\right)\right)
\end{gathered}
$$

Now, we test with $\Delta \vec{v}$ and $\Delta \tau$ and we again insert (3.1)

$$
\begin{gathered}
\frac{1}{2 \operatorname{Pr}} \frac{d}{d t}\|\nabla \vec{v}\|_{2}^{2}+\|\Delta \vec{v}\|_{2}^{2}=\frac{1}{\operatorname{Pr}}(\vec{v} \cdot \nabla \vec{v}, \Delta \vec{v})-\beta\left(e^{-\beta z} \Pi \vec{k}, \Delta \vec{v}\right)-\operatorname{Ra}(\tau \vec{k}, \Delta \vec{v}) . \\
\frac{1}{2} \frac{d}{d t}\|\nabla \tau\|_{2}^{2}+\|\Delta \tau\|_{2}^{2}=(\vec{v} \cdot \nabla \tau, \Delta \tau)-\left(v^{z}, \Delta \tau\right) .
\end{gathered}
$$

Next, we focus on the non-linear terms: for arbitrary positive $\varepsilon$ one can write

$$
\begin{gathered}
(\vec{v} \cdot \nabla \vec{v}, \Delta \vec{v}) \leq \quad\|\vec{v}\|_{4}\|\nabla \vec{v}\|_{4}\|\Delta \vec{v}\|_{2} \leq \frac{1}{\sqrt{2}}\|\vec{v}\|_{2}^{1 / 2}\|\nabla \vec{v}\|_{2}\|\Delta \vec{v}\|_{2}^{3 / 2} \\
\leq \frac{\|\vec{v}\|_{2}^{2}\|\nabla \vec{v}\|_{2}^{4}}{4 \sqrt{2} \varepsilon^{4}}+\frac{3}{4 \sqrt{2}} \varepsilon^{4 / 3}\|\Delta \vec{v}\|_{2}^{2} . \\
(\vec{v} \cdot \nabla \tau, \Delta \tau) \leq \quad\|\vec{v}\|_{4}\|\nabla \tau\|_{4}\|\Delta \tau\|_{2} \leq \frac{1}{\sqrt{2}}\|\vec{v}\|_{2}^{1 / 2}\|\nabla \vec{v}\|_{2}^{1 / 2}\|\nabla \tau\|_{2}^{1 / 2}\|\Delta \tau\|_{2}^{3 / 2} \\
\leq \frac{\|\vec{v}\|_{2}^{2}\|\nabla \vec{v}\|_{2}^{2}\|\nabla \tau\|_{2}^{2}}{4 \sqrt{2} \varepsilon^{4}}+\frac{3}{4 \sqrt{2}} \varepsilon^{4 / 3}\|\Delta \tau\|_{2}^{2} .
\end{gathered}
$$

Let us set

$$
D(t)=\frac{\|\nabla \vec{v}\|_{2}^{2}}{2 \operatorname{Pr}}+\operatorname{Ra} \frac{\|\nabla \tau\|_{2}^{2}}{2}
$$

We can write

$$
\frac{d}{d t} D(t)+\left(1-\frac{3}{4 \sqrt{2} \operatorname{Pr}} \varepsilon^{4 / 3}-\varepsilon^{2}\right)\|\Delta \vec{v}\|_{2}^{2}++\left(1-\frac{3}{4 \sqrt{2}} \varepsilon^{4 / 3}\right) \operatorname{Ra}\|\Delta \tau\|_{2}^{2} \leq \mathbf{C}_{\mathbf{2}}\|\vec{v}\|_{2}^{2} D^{2}(t)+\mathbf{C}_{3} D(t),
$$

where the last two terms on the left hand side are positive provided $\varepsilon$ is sufficiently small, while $\mathbf{C}_{\mathbf{2}}$ depends on $\beta, \operatorname{Pr}, \varepsilon$ and $\mathbf{C}_{3}$ depends on $\operatorname{Ra}, \operatorname{Pr}, \beta, \varepsilon$. 
By (3.5) we know that $\|\vec{v}\|_{2}^{2}$ is uniformly bounded in $[0, T]$ and by (3.6) one also has $D(t) \in$ $L^{2}(0, T)$. As a consequence, by using a generalized Gronwall argument, from

$$
\frac{d}{d t} D(t) \leq \mathbf{C}_{\mathbf{2}} \mathcal{M} D^{2}(t)+\mathbf{C}_{3} D(t):=\mathcal{M}_{2}\left(D^{2}(t)+D(t)\right),
$$

where $\mathcal{M}_{2}$ depends on $\operatorname{Pr}, \operatorname{Ra}, \beta$ and $E(0)$. As we did to get (3.5) we obtain

$$
D(t) \leq \frac{\sqrt{D(0)(D(0)+1)}}{1+D(0)-\sqrt{D(0)(D(0)+1)}}:=\mathcal{M}_{3}(D(0))
$$

We put (3.11) in (3.9), and after integration we also achieve

$$
\int_{0}^{T}\left(\|\Delta \tau\|_{2}^{2}+\|\Delta \vec{v}\|_{2}^{2}\right) d t<\mathbf{C}_{4}\left(\mathcal{M}_{2}^{2}+\mathcal{M}_{2}\right) \log \left[\left(\frac{E(0)+1}{E(0)}\right)\right]^{\frac{1}{2 \mathrm{C}}}+D(0):=\mathcal{M}_{4}
$$

where $\mathbf{C}_{4}$ depends on $\mathrm{Ra}, \operatorname{Pr}, \beta, \varepsilon$ and $E(0)$.

\section{Third a priori estimate}

Here we show that the solution is in fact a Ladyzhenskaya solution. One gets

$$
\begin{gathered}
\vec{v}_{t} \in L^{2}\left((0, T) ; \mathcal{H}_{D}\left(\Omega_{0}\right)\right), \\
\tau_{t} \in L^{2}\left((0, T) ; H_{D}\left(\Omega_{0}\right)\right),
\end{gathered}
$$

which implies, as a consequence

$$
\vec{v} \cdot \nabla \vec{v} \in L^{2}\left(0, T ; L^{2}\left(\Omega_{0}\right)\right) \quad \text { so that } \quad \Pi \in L^{2}\left(0, T ; W_{N}^{1,2}\left(\Omega_{0}\right)\right) .
$$

In order to prove this, we test $(2.1)_{2}$ with $\frac{\partial \vec{v}}{\partial t}$, the non-linear convective term vanishes by the boundary condition. Next, we apply Cauchy-Schwarz and Ladyzhenskaya inequalities (when using (3.1) for the pressure) and get

$$
\left\|\vec{v}_{t}^{2}\right\|_{2} \leq C^{2}\left(\|\Delta \vec{v}\|_{2}^{2}+\|\vec{v}\|_{2}^{2}\|\nabla \vec{v}\|_{2}^{2}+\|\tau\|_{2}^{2}\right) .
$$

where $C$ depends on $\operatorname{Ra}, \operatorname{Pr}$ and $\beta$. Analogously for $\tau_{t}$ we simply have:

$$
\left\|\tau_{t}\right\|_{2}^{2} \leq 2\left(\|\Delta \tau\|_{2}^{2}+\|\vec{v}\|_{2}^{2}\right)
$$

Now, since the norms on the right-hand side are bounded or integrable, we integrate the inequality in $t \in[0, T]$ so getting

$$
\int_{0}^{T}\left(\left\|\vec{v}_{t}\right\|_{2}^{2}+\left\|\tau_{t}\right\|_{2}^{2}\right) d t<C^{2}\left(\mathcal{M}_{4}+\mathcal{M N M}_{1}+\mathcal{M} \log \left(\frac{E(0)+1}{E(0)}\right)^{\frac{1}{2 \mathrm{C}}}\right) .
$$

Finally, we prove two further energy inequalities allowing the existence of a global in time regular and stable solution for Ra and initial data sufficiently small.

Let us formally test $(2.1)$ with $(\vec{v}, \tau)$, then we multiply the third equation by Ra, we use Ladyzenskaya and Poincaré inequalities and sum to get

$$
\frac{1}{2} \frac{d}{d t} E(t)+\|\nabla \vec{v}\|_{2}^{2}+\operatorname{Ra}\|\nabla \tau\|_{2}^{2} \leq \frac{\beta^{2} C^{2}(\beta)}{\operatorname{Pr}^{2}}\|\vec{v}\|_{2}\|\nabla \vec{v}\|_{2}^{2}+\operatorname{Ra}(\beta C(\beta)+2)\left(\frac{\varepsilon^{2}}{2}\|\tau\|_{2}^{2}+\frac{\|\vec{v}\|_{2}^{2}}{2 \varepsilon^{2}}\right) .
$$


In comparison with the first estimate, here we have just changed what comes from the П-term, i.e

$$
\|\vec{v}\|_{4}^{2}\|\vec{v}\|_{2} \leq \frac{1}{\sqrt{2}}\|\vec{v}\|_{2}\|\nabla \vec{v}\|_{2}\|\vec{v}\|_{2} \leq \frac{1}{\sqrt{2}}\|\vec{v}\|_{2}\|\nabla \vec{v}\|_{2}^{2}
$$

At the end, for $\varepsilon \in(0,1)$ we obtain

$$
\frac{d E(t)}{d t}+\left(1-\frac{\beta C(\beta)}{\operatorname{Pr}} \sqrt{E(t)}-\frac{\operatorname{Ra}(\beta C(\beta)+2)}{2 \varepsilon^{2}}\right)\|\nabla \vec{v}\|_{2}^{2}+\operatorname{Ra}\left(1-\frac{\varepsilon^{2}(\beta C(\beta)+2)}{2}\right)\|\nabla \tau\|_{2}^{2} \leq 0,
$$

Now, we can prove

Lemma 3.1. Let the initial data for (2.7) belong to $L^{2}\left(\Omega_{0}\right), \beta<2 \pi, \operatorname{Ra}<\frac{1}{(\beta C(\beta)+2)^{2}}$ and $E(0)<\frac{\operatorname{Pr}^{2}}{16 \beta^{2} C^{2}(\beta)}$, then one has

$$
\begin{gathered}
E(t) \leq e^{-\mathbf{C}_{0} t} E(0), \\
\mathbf{C}_{0} \int_{0}^{+\infty}\left(\|\nabla \vec{v}\|_{2}^{2}+\operatorname{Ra}\|\nabla \tau\|_{2}^{2}\right) d t<E(0) .
\end{gathered}
$$

where $\mathbf{C}_{0}=\min \left\{\frac{\operatorname{Pr}}{2}-2 \beta C(\beta) \sqrt{E(0)}, 1\right\}$.

Proof. By the Poincaré inequality (since $\left.1<5 \pi^{2}\right)$, for $\varepsilon \in(0,1)$, from (3.14) we get

$$
\frac{d E(t)}{d t}+\left(\operatorname{Pr}-\beta C(\beta) \sqrt{E(t)}-\frac{\operatorname{Pr} \operatorname{Ra}(\beta C(\beta)+2)}{2 \varepsilon^{2}}\right) \frac{\|\vec{v}\|_{2}^{2}}{\operatorname{Pr}}+\left(2-\varepsilon^{2}(\beta C(\beta)+2)\right) \frac{\operatorname{Ra}}{2}\|\tau\|_{2}^{2} \leq 0 .
$$

By taking Ra sufficiently small to have

$$
\operatorname{Pr}-\frac{\operatorname{Ra} \operatorname{Pr}(\beta C(\beta)+2)}{2 \varepsilon^{2}}=\frac{\operatorname{Pr}}{2 \varepsilon^{2}}\left(2 \varepsilon^{2}-\operatorname{Ra}(\beta C(\beta)+2)\right)>\frac{\operatorname{Pr}}{2},
$$

we achieve

$$
\frac{d E(t)}{d t}+\left(\frac{\operatorname{Pr}}{2}-2 \beta C(\beta) \sqrt{E(t)}\right) \frac{\|\vec{v}\|_{2}^{2}}{2 \operatorname{Pr}}+\left(2-\varepsilon^{2}(\beta C(\beta)+2)\right) \frac{\operatorname{Ra}}{2}\|\tau\|_{2}^{2} \leq 0 .
$$

Just to fix the ideas, $\varepsilon=1 / \sqrt{(\beta C(\beta)+2)}$ is compatible with all the previous restrictions and implies $2-\varepsilon^{2}(\beta C(\beta)+2)=1$. If moreover

$$
2 \beta C(\beta) \sqrt{E(0)}<\frac{\operatorname{Pr}}{2},
$$

then

$$
C(t):=\min \left\{\frac{\operatorname{Pr}}{2}-2 \beta C(\beta) \sqrt{E(t)}, 1\right\}
$$

is such that $C(0)>0$. Therefore, by coninuity, a maximal $t^{*}>0$ exists such that $C(t)$ is non negative for $t \in\left(0, t^{*}\right)$. Inequality (3.17) now reads

$$
\frac{d E}{d t}+C(t) E(t) \leq 0
$$

and implies that for $t \in\left(0, t^{*}\right)$

$$
E(t) \leq E(0)
$$


By definition if $t^{*}$ is a finite number then $C\left(t^{*}\right)=0$; this means that

$$
\beta C(\beta) \sqrt{E\left(t^{*}\right)}=\frac{\operatorname{Pr}}{2} .
$$

If we put (3.21) in (3.18) we obtain

$$
E\left(t^{*}\right)>E(0),
$$

which is an absurdum since one could at most have $E\left(t^{*}\right)=E(0)$. We have so proved that, since $C(t)$ is bounded from below as follows

$$
0<\frac{\operatorname{Pr}}{2}-2 \beta C(\beta) \sqrt{E(0)} \leq \frac{\operatorname{Pr}}{2}-2 \beta C(\beta) \sqrt{E(t)},
$$

then for all $t>0$

$$
\frac{d E}{d t}+\mathbf{C}_{0} E(t) \leq 0
$$

where $\mathbf{C}_{0}=\mathbf{C}(0)$ is the minimum of $C(t)$. So, the exponential decay follows and can finally be used to integrate $(3.14)$ in $(0, \infty)$ so getting $(3.15)$.

Remark 3.1. If $\beta=0$, which is the classic $O-B$ Bénard problem, the restriction on the initial data $E(0)$ is unnecessary to get the exponential decay.

Remark 3.2. Notice that the rate of decay decreases as $\beta$ increases.

We are now going to prove a last inequality by still making use of a positive arbitrary $\varepsilon$ together with the previous results. In order to do this we need the following:

Remark 3.3. The present boundary conditions imply a Poincaré-like inequality with the gradients bounded by the laplacians. Actually,

$$
\|\nabla \vec{v}\|_{2}^{2}=-(\vec{v}, \Delta \vec{v}) \leq\|\vec{v}\|_{2}\|\Delta \vec{v}\|_{2} \leq\|\nabla \vec{v}\|_{2}\|\Delta \vec{v}\|_{2}
$$

and analogously for $\tau$

$$
\|\nabla \tau\|_{2}^{2}=-(\tau, \Delta \tau) \leq\|\tau\|_{2}\|\Delta \tau\|_{2} \leq \frac{1}{\sqrt{5} \pi}\|\nabla \tau\|_{2}\|\Delta \tau\|_{2} .
$$

Hence, (3.23) and (3.24) are Poincarré inequalities for the gradients.

Lemma 3.2. Let the initial data for (2.7) belong to $W^{1,2}\left(\Omega_{0}\right), \beta<2 \pi$ and $E(0)<\frac{\operatorname{Pr}^{2}}{16 \beta^{2} C^{2}(\beta)}$, then if $\operatorname{Ra}<\min \left\{\frac{1}{(\beta C(\beta)+2)^{2}}, \frac{25 \pi^{4}}{64\left(25 \pi^{4}+1\right)\left(\beta^{2} C^{2}(\beta)+1\right)}\right\}$, it follows that $T^{*}$ and $\mathbf{C}_{6}>0$ exist, only depending on $\operatorname{Pr}$, such that

$$
\begin{gathered}
D(t) \leq e^{\frac{\mathbf{C}_{5}}{\mathbf{C}_{0}} E^{2}(0)} D(0) \\
D(t) \leq e^{-\mathbf{C}_{6} t} D\left(T^{*}\right), \quad \forall t>T^{*}
\end{gathered}
$$

where $\mathbf{C}_{5}$ depends on $\mathrm{Ra}, \operatorname{Pr}$ and $\beta$.

$$
\mathbf{C}_{7} \int_{0}^{+\infty}\left(\|\Delta \vec{v}\|_{2}^{2}+\operatorname{Ra}\|\Delta \tau\|_{2}^{2}\right) d t<D(0)
$$

where $\mathbf{C}_{7}$ depends on $E(0)$, $\operatorname{Pr}, \operatorname{Ra}, \beta, D(0)$ and $T^{*}$. 
Proof. We prove (3.25) by starting from an inequality which is the analogous of (3.9): we get it by just applying Poincaré inequality to the gradients which is possible because of remark 3.3

$$
\begin{aligned}
& \frac{d}{d t} D(t)+\left(1-\frac{3}{4 \sqrt{2} \operatorname{Pr}} \varepsilon^{4 / 3}-\left(\beta^{2} C^{2}(\beta)+1\right) \frac{\mathrm{Ra}}{\varepsilon^{2}}\right)\|\Delta \vec{v}\|_{2}^{2}+\left(1-\frac{3}{4 \sqrt{2}} \varepsilon^{4 / 3}-\left(1+\frac{1}{25 \pi^{4}}\right) \varepsilon^{2}\right) \operatorname{Ra}\|\Delta \tau\|_{2}^{2} \\
& \quad \leq \mathbf{C}_{\mathbf{2}}\|\vec{v}\|_{2}^{2} D^{2}(t) .
\end{aligned}
$$

We first notice that if $\varepsilon<\min \left\{1, \operatorname{Pr}^{3 / 4}\right\}$

$$
\begin{gathered}
1-\frac{3}{4 \sqrt{2} \operatorname{Pr}} \varepsilon^{4 / 3}>\frac{8-3 \sqrt{2}}{8}>\frac{1}{4}, \\
1-\frac{3}{4 \sqrt{2}} \varepsilon^{4 / 3}>\frac{8-3 \sqrt{2}}{8}>\frac{1}{4}
\end{gathered}
$$

and so (3.28) implies also

$$
\frac{d}{d t} D(t)+\left(\frac{1}{4}-\left(\beta^{2} C^{2}(\beta)+1\right) \frac{\mathrm{Ra}}{\varepsilon^{2}}\right)\|\Delta \vec{v}\|_{2}^{2}+\left(\frac{1}{4}-\left(1+\frac{1}{25 \pi^{4}}\right) \varepsilon^{2}\right) \operatorname{Ra}\|\Delta \tau\|_{2}^{2}<\mathbf{C}_{2}\|\vec{v}\|_{2}^{2} D^{2}(t),
$$

Next, if we impose also $\varepsilon^{2}<\frac{25 \pi^{4}}{8\left(25 \pi^{4}+1\right)}$, then $(3.29)$ implies

$$
\frac{d}{d t} D(t)+\left(\frac{1}{4}-\left(\beta^{2} C^{2}(\beta)+1\right) \frac{\mathrm{Ra}}{\varepsilon^{2}}\right)\|\Delta \vec{v}\|_{2}^{2}+\frac{1}{8} \operatorname{Ra}\|\Delta \tau\|_{2}^{2} \leq \mathbf{C}_{\mathbf{2}}\|\vec{v}\|_{2}^{2} D^{2}(t) .
$$

Just for simplicity we choose $\varepsilon^{2}=\frac{25 \pi^{4}}{8\left(25 \pi^{4}+1\right)}$ and so if $\mathrm{Ra}<\frac{25 \pi^{4}}{32\left(25 \pi^{4}+1\right)\left(\beta^{2} C^{2}(\beta)+1\right)}$ and by Lemma 3.1 we get

$$
\frac{d D}{d t}<\mathbf{C}_{2} E(0) D^{2}(t) \quad \forall t \in(0, \infty) .
$$

Then from (3.15) by using the same Gronwall arguments as for (3.12) we have

$$
D(t)<e^{\mathbf{C}_{2} \int_{0}^{\infty} E(0) D(t) d t} D(0)<e^{\frac{\mathbf{C}_{5}}{\mathbf{C}_{0}} E^{2}(0)} D(0) .
$$

Next, since $E(0)$ and Ra satisfy the hypotheses of Lemma 3.1 and since by Remark 3.3 one can use Poincaré inequality on the left-hand side of (3.30), we can write

$$
\frac{d}{d t} D(t)+c_{1}(t)\|\Delta \vec{v}\|_{2}^{2}+c_{2}(t) \operatorname{Ra}\|\Delta \tau\|_{2}^{2}<0
$$

where, with the further restriction $\mathrm{Ra}<\frac{25 \pi^{4}}{64\left(25 \pi^{4}+1\right)\left(\beta^{2} C^{2}(\beta)+1\right)}$

$$
c_{1}(t):=\frac{1}{8}-\mathbf{C}_{2} E(0) D(0) e^{-\mathbf{C}_{0} t}
$$

and

$$
c_{2}(t):=\frac{1}{8}-\mathbf{C}_{2} \operatorname{Pr} E(0) D(0) e^{-\mathbf{C}_{0} t} .
$$

We wish to prove that for sufficiently large $t$ both $c_{1}(t)$ and $c_{2}(t)$ are bounded from below by positive constants.

Since

$$
\lim _{t \rightarrow+\infty} c_{1}(t)=\lim _{t \rightarrow+\infty} c_{2}(t)=\frac{1}{8}
$$


by continuity $T^{*}$ exists such that for $t>T^{*}$ both $c_{1}(t)$ and $c_{2}(t)$ are positive. More,

$$
\sup _{t \in(0, \infty)} c_{1}(t)=\sup _{t \in(0, \infty)} c_{2}(t)=\frac{1}{8}
$$

As a conseguence for $t>T^{*}$ we have the following inequality

$$
\frac{d}{d t} D(t)+\frac{5 \pi^{2}}{8}\left(\|\nabla \vec{v}\|_{2}^{2}+\mathrm{Ra}\|\nabla \tau\|_{2}^{2}\right)<\frac{d}{d t} D(t)+\frac{1}{8}\left(\|\Delta \vec{v}\|_{2}^{2}+\mathrm{Ra}\|\Delta \tau\|_{2}^{2}\right)<0 .
$$

Thus, on one side

$$
D(t) \leq e^{-\mathbf{C}_{6} t} D\left(T^{*}\right),
$$

where $\mathbf{C}_{6}=\frac{5 \pi^{2}}{4} \min \{\operatorname{Pr}, 1\}$; on the other side by integrating from $T^{*}$ to $\infty$

$$
\frac{1}{8} \int_{T^{*}}^{+\infty}\left(\|\Delta \vec{v}\|_{2}^{2}+\operatorname{Ra}\|\Delta \tau\|_{2}^{2}\right) d t<D\left(T^{*}\right)
$$

In $\left(0, T^{*}\right)$ we do not know about the sign of $c_{1}$ and $c_{2}$. However, we come back to (3.30) and use (3.25) (showing the boundedness of $D$ ) together with Lemma 3.1 :

$$
\begin{array}{r}
\int_{0}^{T^{*}} \frac{d}{d t} D(t) d t+\frac{1}{8} \int_{0}^{T^{*}}\left(\|\Delta \vec{v}\|_{2}^{2}+\mathrm{Ra}\|\Delta \tau\|_{2}^{2}\right) d t \leq \mathbf{C}_{2} \int_{0}^{T^{*}}\|\vec{v}\|_{2}^{2} D^{2}(t) d t, \\
D\left(T^{*}\right)+\frac{1}{8} \int_{0}^{T^{*}}\left(\|\Delta \vec{v}\|_{2}^{2}+\mathrm{Ra}\|\Delta \tau\|_{2}^{2}\right) d t \leq \mathbf{C}_{2} \int_{0}^{T^{*}} e^{-\mathbf{C}_{0} t} E(0) c_{4}^{2} D^{2}(0) d t+D(0),
\end{array}
$$

where $c_{4}=e^{\frac{2 \mathbf{C}_{5}}{\mathrm{C}_{0}} E^{2}(0)}$. By summing (3.33) and (3.32) then (3.27) follows.

\section{Existence results for the full non-linear system}

Actually, we apply the operator $\nabla \times$ to (2.1); from the identity $\nabla \times \nabla=0$ we get:

$$
\left\{\begin{array}{l}
\Delta \Pi-\beta \Pi_{z}=-\frac{2 e^{\beta z}}{\operatorname{Pr}}\left[\left(\varphi_{z x}\right)^{2}-\varphi_{x x} \varphi_{z z}\right]+\operatorname{Ra} e^{\beta z} \tau_{z}, \\
\frac{1}{\operatorname{Pr}}\left(\frac{\partial \Delta \varphi}{\partial t}-\operatorname{det}(\nabla \varphi, \nabla \Delta \varphi)\right)-\Delta^{2} \varphi=-\beta e^{-\beta z} \Pi_{x}+\operatorname{Ra} \tau_{x} \\
\frac{\partial \tau}{\partial t}+\operatorname{det}(\nabla \varphi, \nabla \tau)-\Delta \tau=\varphi_{x}
\end{array}\right.
$$

In order to get equivalent problems, the initial conditions

$$
\left\{\begin{array}{l}
\varphi(x, z, 0)=\varphi_{0}(x, z), \\
\tau(x, z, 0)=\tau_{0}(x, z),
\end{array} \quad(x, z) \in \Omega\right.
$$

must be of the kind $\varphi_{0} \in W_{D}^{2,2}\left(\Omega_{0}\right)$ and $\tau_{0} \in W_{N}^{1,2}\left(\Omega_{0}\right)$. The full set of boundary conditions associated to (4.1) becomes:

$$
\left\{\begin{array}{l}
\Pi_{z}(x, 0, t)=\Pi_{z}(x, 1, t)=0, \\
\Delta \varphi(x, 0, t)=\Delta \varphi(x, 1, t)=0, \quad \text { for } \quad(x, t) \in \mathbb{R} \times(0, \infty) . \\
\tau(x, 0, t)=\tau(x, 1, t)=0,
\end{array}\right.
$$


Theorem 4.1. Let $\beta<2 \pi$, Ra and $\operatorname{Pr}$ be arbitrary. If $\vec{v}_{0} \in \mathcal{W}_{D}^{1,2}\left(\Omega_{0}\right)$ and $\tau_{0} \in W_{D}^{1,2}\left(\Omega_{0}\right)$, then a finite $T>0$ exists, such that system (2.1) is fulfilled a.e. in space-time by a unique solution:

$$
\begin{gathered}
\Pi \in L^{1}\left(0, T ; W_{N}^{1,2}\left(\Omega_{0}\right)\right), \\
\vec{v} \in L^{\infty}\left(0, T ; \mathcal{W}_{D}^{1,2}\left(\Omega_{0}\right)\right) \cap L^{2}\left(0, T ; \mathcal{W}_{D}^{2,2}\left(\Omega_{0}\right)\right), \\
\tau \in L^{\infty}\left(0, T ; W_{D}^{1,2}\left(\Omega_{0}\right)\right) \cap L^{2}\left(0, T ; W_{D}^{2,2}\left(\Omega_{0}\right)\right) .
\end{gathered}
$$

If moreover Ra and the initial data $\left\|\vec{v}_{0}\right\|_{2},\left\|\tau_{0}\right\|_{2}$ fulfil the conditions in Lemma 3.2, then the solution exists for all $t$ in the class

$$
\begin{gathered}
\Pi \in L^{1}\left(0, \infty ; W_{N}^{1,2}\left(\Omega_{0}\right)\right), \\
\vec{v} \in L^{\infty}\left(0, \infty ; \mathcal{W}_{D}^{1,2}\left(\Omega_{0}\right)\right) \cap L^{2}\left(0, \infty ; \mathcal{W}_{D}^{2,2}\left(\Omega_{0}\right)\right), \\
\tau \in L^{\infty}\left(0, \infty ; W_{D}^{1,2}\left(\Omega_{0}\right)\right) \cap L^{2}\left(0, \infty ; W_{D}^{2,2}\left(\Omega_{0}\right)\right),
\end{gathered}
$$

and decays exponentially fast in the norm of $W^{1,2}\left(\Omega_{0}\right)$.

Proof. The statement simply follows by proving the existence of a weak solution by the first estimate, then the other properties are an easy consequence of Lemma 3.1 and Lemma 3.3.

For weak solution we mean that $(\Pi, \vec{v}, \tau)$ has to verify

$$
\begin{gathered}
(\nabla \Pi, \nabla \varrho)+\beta\left(\Pi_{z}, \varrho\right)=\frac{1}{\operatorname{Pr}}\left[-\left(e^{\beta z} \vec{v} \cdot \nabla \vec{v}, \nabla \varrho\right)-\beta\left(e^{\beta z} \vec{v} \cdot \nabla v^{z}, \varrho\right)\right]-\operatorname{Ra}\left(e^{\beta z} \tau_{z}, \varrho\right) \\
\frac{1}{\operatorname{Pr}}\left(\frac{\partial \vec{v}}{\partial t}+\vec{v} \cdot \nabla \vec{v}, \vec{\psi}\right)+(\nabla \vec{v}, \nabla \vec{\psi})=-\beta\left(e^{-\beta z} \Pi, \vec{\psi}\right)+\operatorname{Ra}(\tau, \vec{\psi}) \\
\left(\frac{\partial \tau}{\partial t}, \vartheta\right)+(\vec{v} \cdot \nabla \tau, \vartheta)+(\nabla \tau, \nabla \vartheta)=\left(v^{z}, \vartheta\right)
\end{gathered}
$$

for all $\varrho \in W_{N}^{1,2}\left(\Omega_{0}\right), \vec{\psi} \in \mathcal{W}_{D}^{1,2}\left(\Omega_{0}\right)$ and $\vartheta \in W_{D}^{1,2}\left(\Omega_{0}\right)$.

Now, we look for Galerkin approximation solutions, which solve the projection of the system in finite dimension spaces.

We begin the proof by defining such approximation solutions

$$
\begin{gathered}
\tau^{N}=\sum_{i=1,2} \sum_{m, n=1}^{N} A_{m n}^{i} \xi_{m n}^{i}, \\
\Pi^{N}=\sum_{i=1,2} \sum_{m, n=1}^{N} B_{m n}^{i} \phi_{m n}^{i}, \\
\varphi^{N}=-\sum_{i=1,2} \sum_{m, n=1}^{N} \frac{C_{m n}^{i} \xi_{m n}^{i}}{\alpha_{m n}},
\end{gathered}
$$

where the coefficients $A_{m n}^{i}=A_{m n}^{i}(t), B_{m n}=B_{m n}(t), C_{m n}^{i}=C_{m n}^{i}(t)$ are unknowns.

As customary, we define the Galerkin approximation solutions as the solutions of the projection of the PDE system in a finite dimensional subspace (whose dimension is in our case $2 N^{2}$ ), in such a way that the coefficients of the finite sums verify for all values of $i$ and $m, k=1 \ldots N$ an ODE system. 
But in the present case, the system is constrained by the pressure equation which, in order to get a Galerkin solution, has to be also projected, as an algebraic equation, in the same finite subspace where we write the ODE system. To this end we recall the explicit expressions in [3]:

$$
\sum_{n=1}^{N} B_{m n}^{i} \mathcal{D}_{n k}^{m}=-\frac{\beta \mathrm{Ra}}{\alpha_{m k}} \sum_{n=1}^{N} A_{m n}^{i} \mathcal{M}_{n k}+\frac{1}{\operatorname{Pr}} \sum_{i^{\prime} i^{\prime \prime}} \sum_{n, l=1}^{N} C_{r s}^{i^{\prime \prime}} C_{n l}^{i^{\prime}} Q_{r s n l m k}^{i i^{\prime} i^{\prime \prime}}
$$

where

$$
\begin{aligned}
Q_{r s n l m k}^{i i^{\prime} i^{\prime \prime}}= & \left(\frac{2 s \phi_{r s}^{i^{\prime}}}{\alpha_{r s}} \frac{\pi^{2} n l i^{\prime \prime} \phi_{n l}^{-i^{\prime \prime}}}{\alpha_{n l}}-\frac{2 \pi r i^{\prime} \xi_{r s}^{-i^{\prime}}}{\alpha_{r s}} \frac{\xi_{n l}^{i^{\prime \prime}}(\pi l)^{2}}{\alpha_{n l}}, e^{\beta z} i \phi_{m k}^{-i} 2 \pi m\right)- \\
& \left(\frac{\pi s \phi_{r s}^{i}}{\alpha_{r s}} \frac{4 \pi^{2} n^{2} \xi_{n l}^{i^{\prime \prime}}}{\alpha_{n l}}+\frac{2 \pi r i \xi_{r s}^{-i^{\prime}}}{\alpha_{r s}} \frac{2 \pi^{2} n l i^{\prime \prime} \phi_{n l}}{\alpha_{n l}},-e^{\beta z} k \pi \xi_{m k}^{i}+\beta e^{\beta z} \phi_{m k}^{i}\right), \\
\mathcal{D}_{n k}^{m}= & \delta_{n k}+\beta\left\{\begin{array}{cc}
-\frac{2}{\alpha_{m k}}\left(\frac{1}{n+k}+\frac{1}{n-k}\right) n, & \text { if } n+k \text { odd }, \\
0 & \text { if } n+k \text { even, }
\end{array}\right. \\
\mathcal{M}_{n k}= & \pi n\left((-1)^{k+n} e^{\beta}-1\right)\left(\frac{1}{\pi^{2}(n+k)^{2}+\beta^{2}}+\frac{1}{\pi^{2}(n-k)^{2}+\beta^{2}}\right) .
\end{aligned}
$$

Finally for $\beta<2 \pi$, the constraint can be substituted in the Galerkin balance momentum equation in this form:

$$
B_{m j}^{i}=-\frac{\beta \mathrm{Ra}}{\alpha_{m j}}\left(\sum_{l, k=1}^{N} A_{m l}^{i} \mathcal{M}_{l k}+\frac{1}{\operatorname{Pr}} \sum_{i^{\prime} i^{\prime \prime}} \sum_{n, l=1}^{N} C_{r s}^{i^{\prime \prime}} C_{n l}^{i^{\prime}} Q_{r s n l m k}^{i i^{\prime} i^{\prime \prime}}\right)\left(\mathcal{D}^{m}\right)_{k j}^{-1}
$$

By the second equation in (4.1):

$$
\dot{C}_{m j}^{i}+4 \sum_{i^{\prime}, i^{\prime \prime}} \sum_{s, l, r, n=1}^{N} C_{s l}^{i^{\prime}} C_{r n}^{i^{\prime \prime}} G_{r n s l m j}^{i i^{\prime} i i^{\prime \prime}}+\operatorname{Pr} \alpha_{m j} C_{m j}^{i}=2 \pi i m \operatorname{Pr}\left(\beta \sum_{j} \mathcal{N}_{j k} B_{m k}^{-i}-\operatorname{Ra} A_{m j}^{-i}\right)
$$

where

$$
\mathcal{N}_{k j}=\left(1-e^{-\beta}(-1)^{k+j}\right)\left(\frac{\pi(j+k)}{\pi^{2}(j+k)^{2}+\beta^{2}}+\frac{\pi(j-k)}{\pi^{2}(j-k)^{2}+\beta^{2}}\right) .
$$

By the third equation in (4.1) we obtain

$$
\dot{A}_{m j}^{i}+4 \sum_{i^{\prime} i^{\prime \prime}}\left(\sum_{s, l, r, n=1}^{N} A_{s l}^{i^{\prime}} C_{r n}^{i^{\prime \prime}} G_{r n s l m j}^{i i^{\prime} i^{\prime \prime}}\right)+\alpha_{m j} A_{m j}^{i}=-i \frac{C_{m j}^{-i}}{\alpha_{m j}}
$$

where

$$
G_{r n s l m j}^{i i^{\prime} i^{\prime \prime}}=\left(\operatorname{det} J\left(\xi_{r n}^{i}, \xi_{s l}^{i^{\prime}}\right), \xi_{m j}^{i^{\prime \prime}}\right),
$$

The algebraic equations (4.10) leading to the differential equations (4.11) and (4.12) are a constraint for a first order ODE system in normal form. Namely,

$$
\left\{\begin{array}{l}
\dot{\vec{A}}=\vec{C} \cdot \mathbb{A}_{0}(\vec{A})+\mathbb{A}_{1}(\vec{A})+\mathbb{A}_{2}(\vec{C}), \\
\dot{\vec{C}}=\vec{C} \cdot \mathbb{C}_{0}(\vec{C})+\mathbb{C}_{1}(\vec{C})+\mathbb{C}_{2}(\vec{A})+\mathbb{C}_{3}(\vec{B}), \\
\vec{B}=\mathbb{B}_{0}(\vec{A})+\vec{C} \cdot \mathbb{B}_{1}(\vec{C}) .
\end{array}\right.
$$


where $\mathbb{A}_{i}, \mathbb{B}_{i}$ and $\mathbb{C}_{i}$, with $i=0,1,2,3$ are linear operators. Once the expression of $\vec{B}$ is directly substituted in the system, its right hand side is at most quadratic in the "space" variables, so that it can be treated by Peano's Theorem.

Finally, we can give a standard proof that from the sequence of the approximation solutions one can always extract a subsequence weakly converging to a weak solution (4.4). Concerning the linear terms in (4.4), the weak convergence is trivially implied by the uniform bounds in $(3.5),(3.6),(3.11)$ and (3.12), which are fulfilled by the Galerkin solutions too. In fact, they are bounded by the projections of the initial data in the respective finite dimensional spaces.

The nonlinear terms are all convective-like, so that the scheme of the weak convergence can be sketched once for all by writing for instance $(4.4)_{2}$ in the integral form

$$
\frac{1}{\operatorname{Pr}}(\vec{v}(t), \vec{\psi})+\frac{1}{\operatorname{Pr}} \int_{0}^{T}(\vec{v} \cdot \nabla \vec{v}, \vec{\psi}) d t+\int_{0}^{T}(\nabla \vec{v}, \nabla \vec{\psi}) d t=-\beta \int_{0}^{T}\left(e^{-\beta z} \Pi \vec{k}, \vec{\psi}\right) d t+\operatorname{Ra} \int_{0}^{T}(\tau \vec{k}, \vec{\psi}) d t+\left(\vec{v}_{0}, \vec{\psi}\right)
$$

so we want

$$
\int_{0}^{T} \vec{v}_{N} \cdot \nabla \vec{v}_{N} \rightarrow \int_{0}^{T} \vec{v} \cdot \nabla \vec{v}
$$

Since coefficients $C_{m n}^{N i}(t)$, defined in (4.5), are uniformly bounded and uniformly continous, this follows by the convergence to zero a.e. in $t$ of

$$
\left(\left(\vec{v}_{N}-\vec{v}\right) \cdot \nabla \vec{v}_{N}, \vec{\psi}\right)+\left(\vec{v} \cdot \nabla\left(\vec{v}_{N}-\vec{v}\right), \vec{\psi}\right) \leq\left\|\vec{v}_{N}-\vec{v}\right\|_{4}\left\|\nabla \vec{v}_{N}\right\|_{2}\|\vec{\psi}\|_{4}+\left(\vec{v} \otimes \vec{\psi}, \nabla\left(\vec{v}_{N}-\vec{v}\right)\right) .
$$

Since $\vec{v}_{N} \in L^{\infty}\left((0, T), W^{1,2}\left(\Omega_{0}\right)\right), \nabla \vec{v}_{N}$ is bounded because of the a priori estimate and $\vec{\psi} \otimes \vec{v}$ is allowed as test function, being in $L^{\infty}\left((0, T), L^{2}\left(\Omega_{0}\right)\right)$, then the sum tends to zero as $N \rightarrow \infty$.

Remark 4.1. Since the solution in Theorem 4.1 is found as limit of regular divergence free function it is divergence free (as announced). We have so proved that system (2.1) and system (2.7) are equivalent in that class of solutions.

\section{References}

[1] F. Capone, M. Gentile, A.A. Hill, Penetrative convection in a fluid layer with throughflow, Ricerche di Matematica, 57(2):251-260, 2008.

[2] F. Crispo, P. Maremonti, A remark on the partial regularity of a suitable weak solution to the Navier-Stokes Cauchy problem, Discrete and Continuous Dynamical Systems, 37 (3) : 1283-1294, 2015.

[3] A. Corli, A. Passerini, The Bénard problem for slightly compressible materials: existence and linear instability, Mediterranean Journal of Mathematics 16(1), 2018.

[4] P. Falsaperla, G. Mulone Stability in the rotating Bénard problem with Newton-Robin and fixed heat flux boundary conditions, Mechanics Research Communications, 37(1): 122-128, 2010.

[5] G.P. Galdi, An introduction to the mathematical Theory of the Navier-Stokes equation. Vol. I and II, Springer, New York, 1998.

[6] G.P. Galdi, Non-linear stability of the magnetic Bénard problem via a generalized energy method, Archive for Rational Mechanics and Analysis, 87: 167-186, 1985. 
[7] G.P Galdi, M. Padula New approach to energy theory in the stability of fluid motion, Archive for Rational Mechanics and Analysis, 110: 187-286, 1990.

[8] A. Giacobbe, G. Mulone, Stability in the Rotating Benard Problem and Its Optimal Lyapunov Functions, Acta Applicandae Mathematicae 132(1), 2014.

[9] H. Gouin, T. Ruggeri, A consistent thermodinamical model of incompressible media as limit case of quasi-thermal-incopressible materials, Int. J Non-linear Mech., 47:688693,2012 .

[10] Y. Kagei, M. Ruzicka, The Oberbeck-Boussinesq approximation as a constituitive limit, Contin. Mech. Thermodyn, 28(5):1411-1419,2016.

[11] A. Lamacz, A. Passerini, G. Thäter, Natural convection in horizontal annuli: evaluation of the error for two approximations, GEM- International Journal on Geomatematics 2 (2), 307, 2011.

[12] P. Maremonti, A note on the Prodi-Serrin conditions for the regularity of a weak solution to the Navier-Stokes equations, Journal of Mathematical Fluid Mechanics,20(2): 379-392, 2018.

[13] P. Maremonti, S. Shimizu, Global Existence of Solutions to 2-D Navier-Stokes Flow with Non-decaying Initial Data in Exterior Domains, Journal of Mathematical Fluid Mechanics, 20(n02):1-29, 2017.

[14] A. Passerini, Further results on the Oberbeck-Boussinesq convection of an almost compressible fluid (in preparation).

[15] A. Passerini, C. Ferrario A theoretical study of the first transition for the non-linear Stokes problem in a horizontal annulus, International Journal of Non-Linear Mechanics 78, 2015.

[16] A. Passerini, T. Ruggeri, The Bénard problem for quasi-thermal-incompressible materials: a linear analysis, International Journal of Non-Linear Mechanics 67, No 1, pp: 178-185, 2014.

[17] A. Passerini, M. Ruzicka, G. Thäter, Natural convection between two horizontal coaxial cylinders, Zeitschrift fur angewandte mathematik und mechanik 89, No. 5, pp: 399-413, 2009 .

[18] A. Passerini, C. Ferrario, M. Ruzicka, G. Thäter, Theoretical Results on Steady Convective Flows between Horizontal Coaxial Cylinders, SIAM Journal on Applied Mathematics, 71(2): 465-486, 2011. 